\title{
Short term improvement of migraine headaches during ketogenic diet: a prospective observational study in a dietician clinical setting
}

\author{
C Di Lorenzo ${ }^{1 *}$, G Coppola ${ }^{2 *}, G$ Sirianni $^{3}$, F Pierelli $^{1}$ \\ From The European Headache and Migraine Trust International Congress \\ London, UK. 20-23 September 2012
}

\section{Introduction}

Migraine prophylaxys is an important clinical challenge, sometime complicated by side effects. Among that weight increase is one of most frequent.

\section{Background}

Ketogenic diets (KDs), by drastic carbohydrate restriction, induces lipidic metabolism and Ketone bodies synthesis. Other than epilepsy, KDs were already suggested to be effective also in migraine (although in lack of definitive evidences) and in weight loss[1]. We have evaluated if headache and analgesics consumption improved in migraineurs self referred to a dietician, comparing followers of $\mathrm{KD}$ and followers of standard low-calories diet (SD).

\section{Methods}

Migraineurs were found and enrolled in a dietician clinical setting. All clinical data were recorded before the diet initiation and, blind to neurologist, subjects were divided in two subgroups: KD and SD followers. After a one month period of diet, patients were re-evaluated for comparisons.

\section{Results}

Headache frequency and drug consumption was reduced during the observation period, but only in KD group. Responder rates in KD group (52 subjects) were higher than $90 \%$ in terms of attack frequency and drug consumption in the month of observation, while SD group (56 subjects) has no effect.

\section{Conclusion}

KD ameliorates headache and reduces drug consumption in migraineurs, while the SD is fully ineffective on migraine in a short term observation. Our findings support the role of KDs in migraine treatment, maybe modulated by KBs inhibitory effects on neural inflammation and cortical spreading depression [2], and enhancing brain mitochondrial metabolism [3]. Ketogenic VLCD could find a transient role in antagonize the ponderal increase, a common side effect among prophylactic migraine treatments.

\section{Conflict of interest}

none.

Author details

${ }^{1}$ Sapienza University of Rome, Italy. ${ }^{2}$ G.B. Bietti Foundation-IRCCS, Dept of

Neurophysiology of Vision and Neurophthalmology, Rome, Italy.

${ }^{3}$ Wellness and Dietary Medicine, Krom Genetics Institute, Rome, Italy.

Published: 21 February 2013

\section{References}

1. Maggioni F, Margoni M, Zanchin G: Ketogenic diet in migraine treatment: a brief but ancient history. Cephalalgia 2011, 31:1150-1151.

2. de Almeida Rabello Oliveira M, da Rocha Ataíde T, et al: Effects of shortterm and long-term treatment with medium- and long-chain triglycerides ketogenic diet on cortical spreading depression in young rats. Neurosci Lett 2008, 434:66-70.

3. DeVivo DC, Leckie MP, Ferrendelli JS, McDougal DB Jr: Chronic ketosis and cerebral metabolism. Ann Neurol 1978, 3:331-337.

doi:10.1186/1129-2377-14-S1-P219

Cite this article as: Di Lorenzo et al:: Short term improvement of migraine headaches during ketogenic diet: a prospective observational study in a dietician clinical setting. The Journal of Headache and Pain 2013 14(Suppl 1):P219.

'Sapienza University of Rome, Italy

${ }^{2}$ G.B. Bietti Foundation-IRCCS, Dept of Neurophysiology of Vision and

Neurophthalmology, Rome, Italy

Full list of author information is available at the end of the article 